\title{
Synchronous Quadruple Colon Cancer With Two Lesions Previously Obscured by Ischemic Colitis, Plus Bladder Cancer and Thymoma: A Case Report
}

\author{
Byung-Soo Park ${ }^{1}$, Sung Hwan Cho ${ }^{1}$, Su Jin Kim², Tae Un Kim³ ${ }^{3}$ Dong Il Kim', Gyung Mo Son ${ }^{1}$, \\ Hyun Sung Kim ${ }^{1}$ \\ ${ }^{1}$ Department of Surgery, ${ }^{2}$ Division of Gastroenterology, Department of Internal Medicine, ${ }^{3}$ Department of Radiology, Pusan National \\ University Yangsan Hospital, Pusan National University School of Medicine, Yangsan, Korea
}

Synchronous quadruple colorectal cancer (CRC) is extremely rare without genetic alterations. We present a case of synchronous quadruple CRC with 2 lesions previously obscured by ischemic colitis. A 73-year-old woman was admitted to our emergency department. An abdominal computed tomography revealed ischemic colitis and irregular wall thickening of the sigmoid colon and sigmoid-descending junction, suspicious of 2 colon cancers. A colonoscopy examination revealed a fungating mass $20 \mathrm{~cm}$ from the anal verge, as well as ischemic colitis spanning the mucosa from the sigmoid colon to the transverse colon. The patient underwent laparoscopic Hartmann procedure. Pathologic examination confirmed both lesions as adenocarcinomas with microsatellite stable. Seven months postoperatively, instead of a laparoscopic Hartmann reversal, a laparoscopic total colectomy was performed due to the continued presence of severe ischemic colitis. The pathologic report suggested the presence of 2 distinct invasive adenocarcinomas in the descending colon without genetic alterations such as microsatellite instability.

Keywords: Colorectal neoplasms; Quadruple; Ischemic colitis

\section{INTRODUCTION}

Colorectal cancer (CRC) is one of the most common gastrointestinal cancers worldwide [1]. Synchronous colorectal cancer (sCRC) is not uncommon, with an incidence rate of approximately $3.5 \%$ of all colorectal carcinomas (1.1\% to $8.1 \%$ ) [2-4]. Risk factors for sCRC include advanced age, male sex, genetic predisposition to familial adenomatous polyposis (FAP), hereditary nonpolyposis colorectal cancer (HNPCC), and ulcerative colitis $[2,4,5]$. Although sCRC is not uncommon, synchronous

Received: Mar 6, 2020 - Revised: Jun 8, 2020 • Accepted: Jun 18, 2020 Correspondence to: Hyun Sung Kim, M.D.

Department of Surgery, Pusan National University Yangsan Hospital, 20 Geumo-ro, Mulgeum-eup, Yangsan 50612, Korea

Tel: +82-55-360-2124, Fax: +82-55-360-2154

E-mail: gustohs@pusan.ac.kr

ORCID: https://orcid.org/0000-0002-7607-4597

(C) 2021 The Korean Society of Coloproctology

This is an open-access article distributed under the terms of the Creative Commons Attribution NonCommercial License (https://creativecommons.org/licenses/by-nc/4.0) which permits unrestricted non-

commercial use, distribution, and reproduction in any medium, provided the original work is properly cited. quadruple CRC is very rare, especially in patients who do not have the aforementioned risk factors. Furthermore, it is also extremely rare that 2 malignant masses are masked by ischemic colitis.

Herein, we present a case of synchronous quadruple CRC lesions, 2 of which were previously obscured by ischemic colitis lesions and successfully treated. This study was approved by the Institutional Review Board of the Pusan National University Yangsan Hospital (No. 05-2020-040). The requirement for informed consent was waived.

\section{CASE REPORT}

A 73-year-old woman with suspected mechanical bowel obstruction was admitted to our emergency department with a one-day history of abdominal pain and vomiting. One month prior to admission, the patient had received inpatient treatment at a different center for acute hemorrhagic gastritis. The hemoglobin level at the previous admission had been approximately $3 \mathrm{~g} / \mathrm{dL}$. She had a history of hypertension and diabetes. There was no family history 
of cancer. Physical examination revealed generalized abdominal pain and tenderness; no rebound tenderness was reported. Blood pressure at admission was $130 / 80 \mathrm{mmHg}$, pulse rate was 100 beats/minute, and respiratory rate was 16 breaths/minute. Recorded temperature was $36.3^{\circ} \mathrm{C}$, and peripheral capillary oxygen saturation level was $99 \%$. Laboratory tests upon admission revealed white blood cell count of $33.19 \times 10^{3} / \mu \mathrm{L}$, platelet count of $372 \times 10^{3} / \mu \mathrm{L}$, hemoglobin level of $12.5 \mathrm{~g} / \mathrm{dL}$, and C-reactive protein value of $28.87 \mathrm{mg} / \mathrm{L}$. The blood amylase and lipase levels were $26 \mathrm{U} / \mathrm{L}$ and $11 \mathrm{U} / \mathrm{L}$, respectively.

An abdominal computed tomography (CT) scan revealed ischemic colitis with irregular bowel wall thickening and enhancement of the sigmoid colon and sigmoid-descending (SD) junction, suspicious of 2 colon cancers (Fig. 1). The CT also revealed a polypoid mass $(1.4 \mathrm{~cm})$ in the bladder. The colonoscopy was performed after bowel preparation with $4 \mathrm{~L}$ of a polyethyleneglycolelectrolyte solution (Colonlyte, Taejoon Pharm, Seoul, Korea).


Fig. 1. Computed tomography scan of the abdomen and pelvis. (A) Irregular bowel wall thickening and enhancement of the sigmoid colon (arrow). (B) Irregular bowel wall thickening and enhancement of the sigmoid-descending junction (arrow).
The colonoscopy examination showed a fungating mass $20 \mathrm{~cm}$ from the anal verge, causing a partial obstruction, so the scope could be inserted up to the level of the hepatic flexure. We did not identify any masses in the SD junction. Alterations consistent with ischemic colitis, such as submucosal hemorrhage and ulcer-

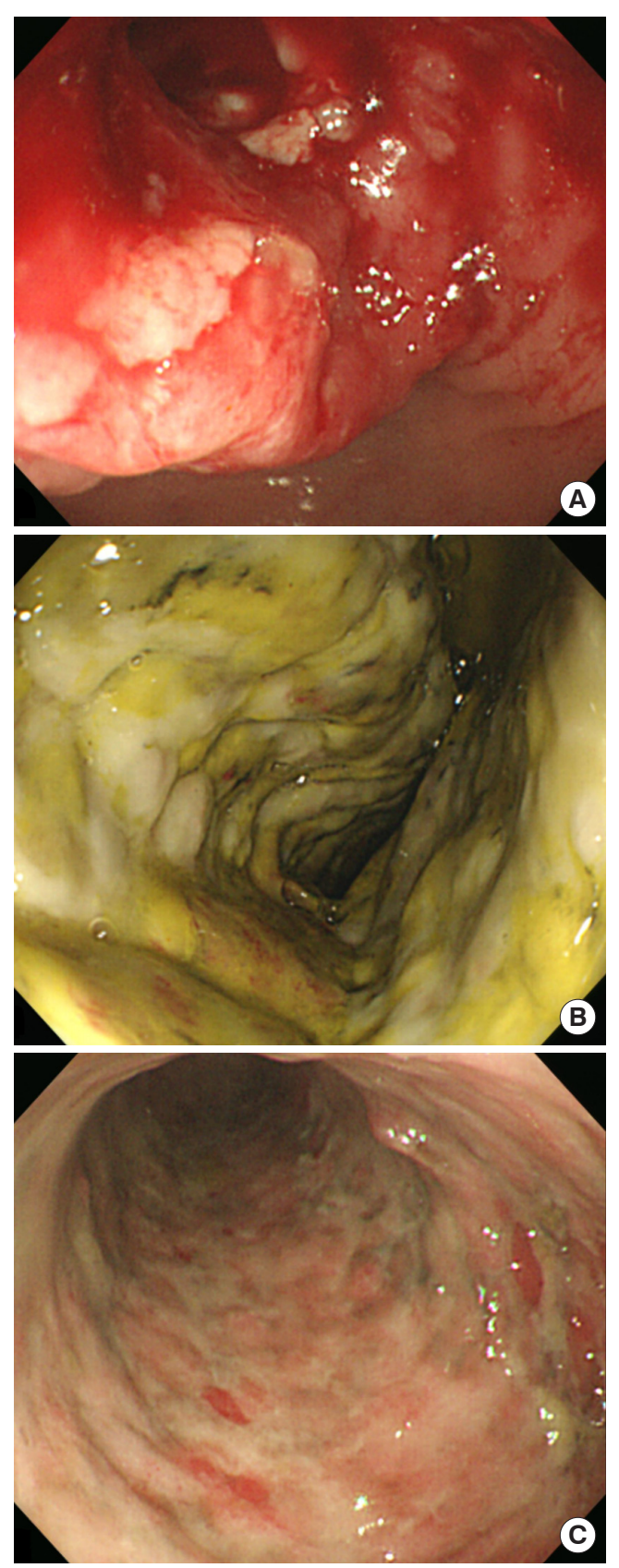

Fig. 2. Colonoscopy images. (A) Fungating mass surrounding the lumen $20 \mathrm{~cm}$ from the anal verge. (B) Ischemic disease, submucosal hemorrhage, and ulceration in the sigmoid colon and transverse colon. (C) Remnant ischemic colitis despite improvement in the preoperative colonoscopy. 
ation, were observed across the mucosa from the sigmoid colon to the transverse colon (Fig. 2). The mass was histologically confirmed to be adenocarcinoma. Preoperative staging with chest CT showed no metastasis. However, thymoma was suspected due to the presence of a soft tissue mass located in the anterior mediastinum (Fig. 3). Positron emission tomography (PET)/CT scan revealed focal fluoro-deoxyglucose uptake in the sigmoid colon and SD junction, as well as in the descending colon, consistent with ischemic colitis (Fig. 3).

Twelve days after visiting the hospital, preoperative repeated colonoscopy was performed up to the level of cecum, which suggested an overall improvement of ischemic colitis; however, there remained remnant colitis of 20 to $40 \mathrm{~cm}$ from the anal verge (Fig. 2). The patient underwent laparoscopic Hartmann operation of the sigmoid colon 18 days after the visit. During the procedure,
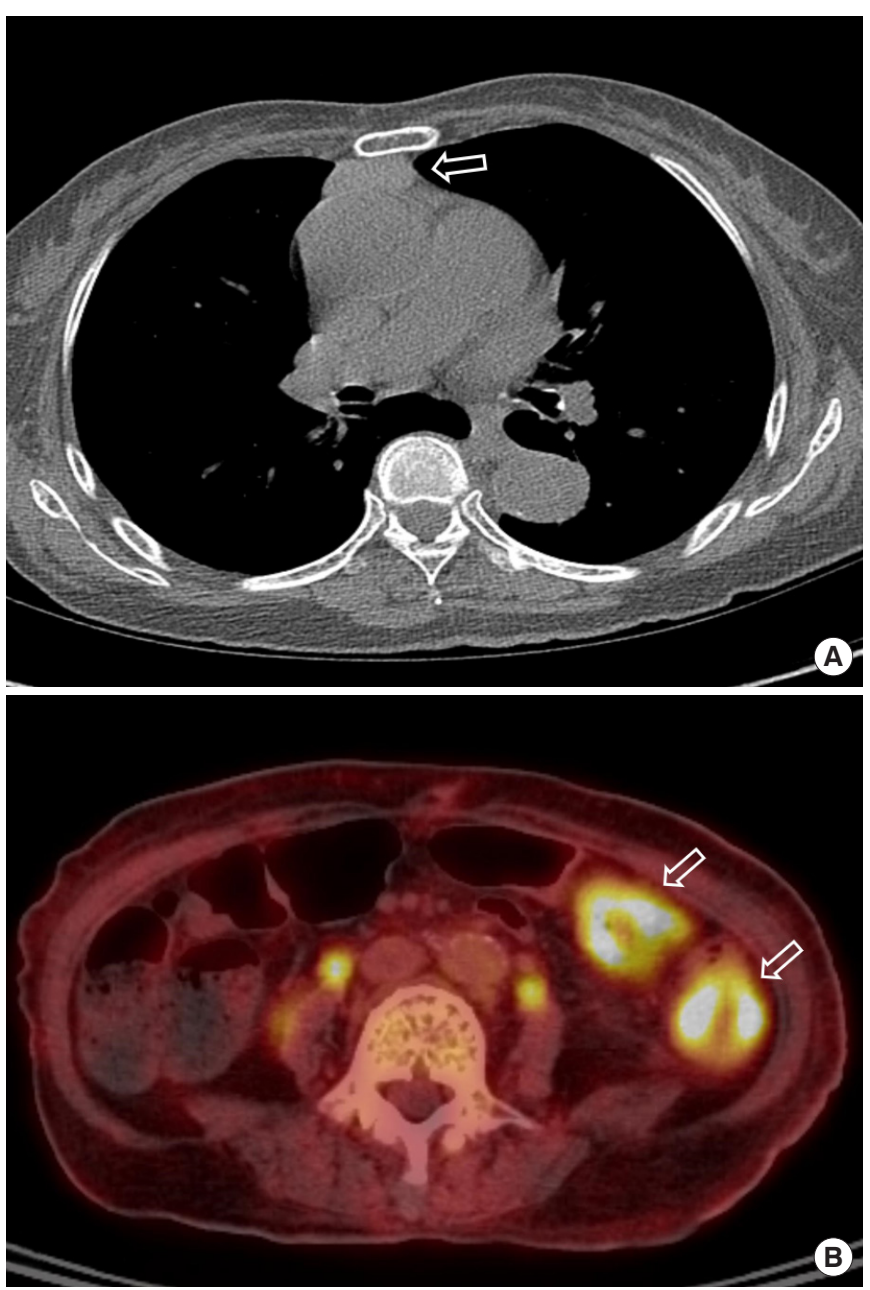

Fig. 3. Computed tomography (CT) scan of the chest and positron emission tomography/CT scan. (A) Soft tissue mass in the anterior mediastinum (arrow). (B) Focal fluoro-deoxyglucose uptake in the sigmoid colon and the sigmoid-descending junction (arrows). apart from the cancer of the sigmoid colon, an additional distinct lesion suspicious for malignancy was found in the SD junction. The patient then underwent radical resection with a D3 lymph node (LN) dissection. Bowel anastomosis was not possible due to severe ischemic colitis; therefore, the patient underwent end colostomy instead. A transurethral resection of the bladder was also performed due to bladder cancer. There were no complications, and the patient was discharged on postoperative day (POD) 10. Pathological examination confirmed that both lesions were adenocarcinomas. The size of the sigmoid colon cancer was $6.5 \mathrm{~cm}$, whereas that of the SD junction cancer was $4.6 \mathrm{~cm}$. Both masses were staged as pT3N0M0, pStageIIA, perineural invasion-positive. Forty-eight LNs were harvested, and there was no evidence of LN metastasis. Microsatellite instability analyses were performed using 5 primers (D5S346, Bat26, Bat25, D17S250, and D2S123). The test showed microsatellite stable (MSS) status. KRAS mutations were not detected. Chemotherapy was recommended; however, the patient refused it due to advanced age and compromised physical conditions. The bladder cancer was confirmed to be a noninvasive papillary urothelial carcinoma confined to the epithelium. The patient was recommended to consult a thoracic surgeon for the evaluation and management of the mass in her chest, suspicious of thymoma. However, she refused further evaluation.

The patient underwent a follow-up CT 7 months after the operation, and no evidence of local tumor recurrence was detected. A follow-up colonoscopy was also attempted but could not be completed due to stomal stenosis. Moreover, laparoscopic Hartmann reversal was planned; however, evidence of severe ischemic colitis remained in the surgical view. Therefore, we explained operation methods to the patient's guardians, and they consented to total colectomy as a definite procedure because colitis persisted at the time, and there was a possibility of colitis recurring in the remnant colon afterward. Eventually, the patient underwent laparoscopic total colectomy with ileorectal anastomosis. No mass lesions were observed intraoperatively, but the pathology report suggested the presence of 2 distinct invasive adenocarcinomas in the descending colon, $2.5 \mathrm{~cm}$ and $3.5 \mathrm{~cm}$ in size. Both masses were staged as pT3. Seventy-seven LNs were harvested, and none presented LN metastases. The tumors were also MSS tumors. KRAS mutations were not detected as well. Finally, she did not have any known genetic tendencies for colon cancer, including FAP or HNPCC. There were no complications, and the patient was discharged on POD 10. Sigmoidoscopy was performed 3 months after the operation and did not show any new lesions.

\section{DISCUSSION}

The sCRC is defined as 2 or more primary colorectal malignant lesions found simultaneously or within 6 months $[1,5]$. If the tumors are found more than 6 months apart, the condition is referred to as metachronous CRC [3]. In the case presented here, 2 
tumors were found 7 months after the first 2 colorectal lesions were diagnosed. Nevertheless, we can safely assume that these 2 colorectal masses had existed previously but had been obscured by ischemic colitis. An initial colonoscopy at the site of the latter 2 lesions had previously resulted in a diagnosis of ischemic colitis, and both of these lesions were later revealed as pT3. It can easily be assumed that the progression of CRC from genesis to stage T3 requires at least 1 month. Furthermore, 1 of the 2 lesions found during the initial surgery may have also been obscured by ischemic colitis during the colonoscopy, further supporting our assumption. Therefore, we considered this case to be an sCRC.

The sCRC is not uncommon; the reported incidence rates range between $1.1 \%$ and $8.1 \%$ of all CRC cases [2-4]. However, synchronous quadruple CRC is extremely rare. Risk factors for sCRC include age, sex, FAP, HNPCC, and ulcerative colitis [2-5]. A recent study has shown a higher incidence of sCRC in patients with hypertension and liver cirrhosis; however, whether common mechanisms and pathways are involved in these diseases has not been established [5]. In the case presented here, well-known genetic alterations related to colon malignancy such as FAP and HNPCC or ulcerative colitis were not detected. The lack of previous risk factors, combined with findings that the 2 colorectal tumors had previously been masked by ischemic colitis, makes this case noteworthy. Based on our findings, we recommend that in cases of ischemic colitis, patients should undergo frequent follow-up with colonoscopy to eliminate the possibility of undetected colon cancer. This is supported by a result in which about $5 \%$ of patients with ischemic colitis had a colon obstruction, and half of these patients had colon cancer [6]. Furthermore, when colorectal surgeons perform surgeries for colon cancer with ischemic colitis, they should check carefully for the possibility of sCRC before and during the surgery.

Since sCRC is not uncommon, a preoperative full colonoscopy should be performed in patients diagnosed with CRC. In cases where this is impossible due to cancer-related bowel lumen stenosis, a full colonoscopy should be repeated as soon as possible after surgery $[4,5]$. PET/CT scans aids in the diagnosis of sCRC in cases of stenosis related to cancer $[1,4]$. However, it should also be noted that $\mathrm{CRC}$ can be masked by other lesions such as ischemic colitis in PET/CT scans, as noted in the present case.

The primary treatment for sCRC is surgical resection. However, the optimal extent of surgical intervention has not yet been established. Some doctors are in favor of a total or subtotal colectomy, while others argue that segmental radical resection with frequent follow-up would reduce morbidity and increase the postoperative quality of life $[1,2,7]$. Thus, the extent of the colon resection for sCRC in the absence of hereditary colorectal malignancy should be determined after consideration of many factors such as the number and location of cancers, accompanying colonic disease, availability of frequent colonoscopy, or patients' performance status. The reported prognosis of sCRC patients varies widely, possibly due to the heterogeneity in the sCRC with clinicopathological characteristics and multifactorial etiology $[1,2,4,5]$.

In conclusion, it is recommended that patients diagnosed with CRC should undergo further examination for sCRC using colonoscopy. Additionally, patients diagnosed with ischemic colitis should be referred for thorough biopsy or early follow-up colonoscopy to eliminate the possibility of undetected colon cancer.

\section{CONFLICT OF INTEREST}

No potential conflict of interest relevant to this article was reported.

\section{REFERENCES}

1. Yang J, Peng JY, Chen W. Synchronous colorectal cancers: a review of clinical features, diagnosis, treatment, and prognosis. Dig Surg 2011;28:379-85.

2. Lam AK, Chan SS, Leung M. Synchronous colorectal cancer: clinical, pathological and molecular implications. World J Gastroenterol 2014;20:6815-20.

3. Tziris N, Dokmetzioglou J, Giannoulis K, Kesisoglou I, Sapalidis $\mathrm{K}$, Kotidis E, et al. Synchronous and metachronous adenocarcinomas of the large intestine. Hippokratia 2008;12:150-2.

4. Kato T, Alonso S, Muto Y, Noda H, Miyakura Y, Suzuki K, et al. Clinical characteristics of synchronous colorectal cancers in Japan. World J Surg Oncol 2016;14:272.

5. Chin CC, Kuo YH, Chiang JM. Synchronous colorectal carcinoma: predisposing factors and characteristics. Colorectal Dis 2019; 21:432-40.

6. Chiu HH, Chen CM, Mo LR, Chao TJ. Gastrointestinal: ischemic colitis associated with colon cancer. J Gastroenterol Hepatol 2005; 20:1458.

7. Yeh CC, Hsi SC, Chuu CP, Kao YH. Synchronous triple carcinoma of the colon and rectum. World J Surg Oncol 2013;11:66. 